PROC. OF JSCE,

No. 235, MARCH 1975

\title{
ELASTO-PLASTIC BEHAVIOR OF THIN-WALLED STEEL TUBES UNDER COMBINED FORCES
}

\author{
By Sadao KomatsU* and Tatsuro SAKIмото**
}

\section{SYNOPSIS}

An incremental force-deformation relationship under combined forces including uniform torsion is derived about thin-walled closed cross-section based on the flow theory of plasticity and von Mieses yield criterion. Thin-walled tubes of welded box cross-sections were tested under combined forces of axial compression and torsion. Some influences of residual stresses and loading path on the inelastic torsional behavior of compression members are discussed and the theoretical predictions are shown to be in good agreement with the test results. On the basis of these discussions, comments on the contradictions between the flow theory and the deformation theory of plasticity, appeared in the inelastic buckling problem, are presented.

\section{INTRODUCTION}

Shear moduli of structural materials stressed beyond the proportional limit have aroused special interest of many investigators, and their evaluation has been regarded as an fundamental matter in various structural problems. This problem appeared, at first, in the experimental studies ${ }^{1) \sim 3}$ which were performed to verify the validity of the mathematical theories of plasticity, often called flow or deformation theories). These tests were carried out with small test specimens in uniform plastic state. The differences between these theories of plasticity were very difficult to examine and appeared slightly only when the loading varies abruptly its path after the specimen overstrained into deep plasticity. In addition to the agreement of the theoretical

* Dr. Eng., Professor of Civil Engineering, Osaka University.

** M. Eng., Lecturer of Applied Mechanics. Kumamoto University. estimation with the experimental results, by reason of mathematical consistency and physical validity ${ }^{5}$, the flow theory of plasticity are considered to be rather effective than the deformation theory of plasticity.

Besides these experimental results, many investigators attempted to determine the magnitude of inelastic shear modulus only at the beginning of inelastic buckling, which was considered as the bifurcation problem. For example, Bijlaard ${ }^{(6), 7)}$ used the deformation theory, Haaijer ${ }^{8)}$ tried to estimate it both by the flow theory and by local buckling tests, while Lay9) used slip theory. In these studies, the discrepancies between the theoretical estimations and Haaijer's experimental results are regarded as caused by the existence of shear stress and initial imperfections. Detailed discussion is reported in Ref. 9. On the other hand, Neal ${ }^{11)}$ carried out experiments about the elasto-plastic lateral instability of mild steel beams and examined that the initial torsional rigidity remains unaltered at its elastic value under constant planar bending when partial yielding has occured. This fact is to be necessarily predicted by the flow theory of plasticity ${ }^{10}$.

In order to analyze not only above mentioned buckling problems, but also inelastic behavior of space structures or planar structures with initial imperfections, the force-deformation relationships of a member with partially yielded cross-section need to be established. Particular$1 y$, the absense of precice estimation of torsional rigidity of partially yielded cross-section seems to hamper the theoretical developments in these fields ${ }^{12}$. Hill and Siebel ${ }^{13), 14)}$ tested thin-walled circular tubes under proportional loading of combined bending and torsion from elastic state to fully plastic state and also showed that the experimental force-deformation curves coincide with the prediction of the flow theory of plasticity very well.

The purpose of this investigation is to examine the torsional behavior of structural steel mem- 
bers with residual stress due to welding in the elasto-plastic range under interaction of combined forces. Thin-walled tubes of welded box crosssection and thin-walled circular tubes without residual stress were tested under two sorts of loading path, one of which is proportional loading and the other is torsion under constant compression. Attention was mainly paid to the direction of deformation vector, initial and successive torsional rigidity, the yield criterion, the influence of loading path on the torsional rigidity and so on. Some influences of the residual stress on the inelastic torsional behavior of compression members are discussed and the theoretical prediction of the flow theory of plasticity are shown to be in good agreement with the experimental results.

\section{THEORETICAL STUDY}

\section{(1) Assumptions and Approximations}

It is much important to make clear the incremental force-deformation relationship for the cross section subjected to axial force, bending moment as well as uniform torsion. Such kind of subject is discussed under the following assumptions and limitations:

1) The cross section is thin-walled, closed and doubly symmetric one.

2) The material has the elastic-perfectly plastic stress-strain curve and the yield criterion of von Mieses is valid in it.

3) The stress-strain relation of Prandtl-Reuss is valid in the plastic range.

4) The cross sectional shape is such that warping torsion may be disregarded and St. Venant torsion is dominant.

5) The shear flow due to St. Venant torsion must be distributed uniformly in the cross section at any stress state.

6) The deformation is small and its additional influences on the equilibrium are ignored*.

\section{(2) Derivation of Tangent Stiffness Matrix}

Consider a partially yielded cross-section subjected to combined forces as shown in Fig. 1. The coordinates $x, y, z$ of a member, which form a right-handed system, originate from an arbitrary fixed point $O$, which is, for example, the

* Influences of additional torsion due to twist of the longitudinal fibers may not be negligible for an open cross section as reported in recent paper of Ref. 15.

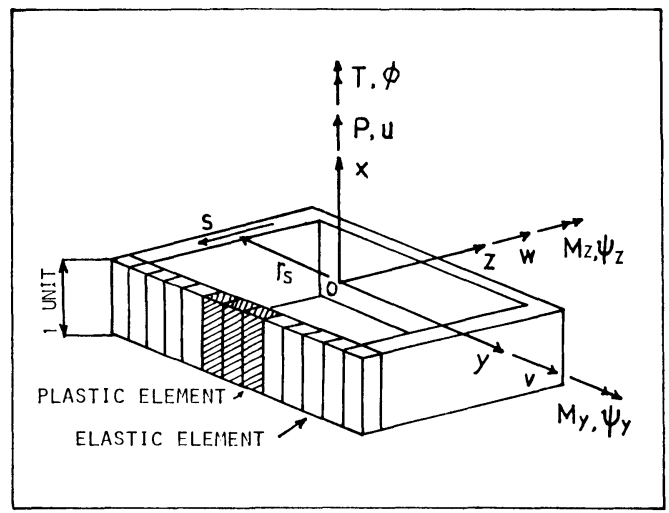

Fig. 1 Positive Vectors of Forces and Deformations.

centroid of the cross section. Normal strain $\varepsilon$ and shear strain $\gamma$ at any point in the cross section are given by

$$
\begin{aligned}
& \varepsilon=\frac{\partial u}{\partial x}-\left(\frac{\partial^{2} v}{\partial x^{2}}\right) \cdot y-\left(\frac{\partial^{2} w}{\partial x^{2}}\right) \cdot z+\varepsilon_{r} \\
& \gamma=\frac{\partial \omega}{\partial s}+\frac{\partial\left(r_{s} \phi\right)}{\partial x} \quad \ldots \ldots \ldots \ldots \ldots \ldots \ldots \ldots \ldots \ldots \ldots \ldots
\end{aligned}
$$

in which $u=$ displacement of the point $\mathrm{O}$ in the $x$ direction; $v, w=$ displacements of the section's shear center in the $y$ and $z$ direction; $\varepsilon_{r}=$ residual strain; $\omega=$ warping of the cross section; $s=$ curvilinear coordinate along the component plates of the cross section; $r_{s}=$ normal distance from the section's shear center to a tangent drawn at any point of the cross section; and $\phi$ $=$ rotation of the cross section. Accordingly, the strain increments are expressed by the increments of the deformations as follows:

$$
\begin{aligned}
& \Delta \varepsilon=\Delta \varepsilon_{0}-\Delta \Psi_{z} \cdot y+\Delta \Psi_{y} \cdot z \\
& \Delta \gamma=\frac{\partial \Delta \omega}{\partial s}+r_{s} \cdot \Delta \theta \quad \ldots \ldots \ldots
\end{aligned}
$$

in which the symbols with $\Delta$ denote the increment of each values and

$$
\left.\begin{array}{ll}
\Delta \varepsilon_{0}=\frac{\partial \Delta u}{\partial x}, & \Delta \Psi_{z}=\frac{\partial^{2} \Delta v}{\partial x^{2}}, \\
\Delta \Psi_{v}=-\frac{\partial^{2} \Delta w}{\partial x^{2}}, & \Delta \theta=\frac{\partial \phi}{\partial x} .
\end{array}\right\}
$$

The incremental stress-strain relations in the plastic range are given from the equation of Prandtl-Reuss and the yield criterion of von Mieses. That is ${ }^{10)}$,

$$
\begin{aligned}
& \Delta \gamma=\frac{\Delta \tau}{G}+\frac{3 \tau}{\sigma}\left(\Delta \varepsilon-\frac{\Delta \sigma}{E}\right) \\
& \Delta \sigma=-\frac{3 \tau}{\sigma} \cdot \Delta \tau \quad \cdots \cdots \cdots
\end{aligned}
$$


and in the elastic range,

$$
\begin{aligned}
\Delta \gamma & =\frac{\Delta \tau}{G} \\
\Delta \sigma & =E \cdot \Delta \varepsilon
\end{aligned}
$$

in which $G=$ shear modulus; $\tau=$ shear stress; $\sigma$ =normal stress; and $E=$ Young's modulus. Integrating Eq. (4) around the whole cross section and substituting Eq. (6) for the plastic zone and Eq. ( 8 ) for the elastic zone yield

$$
\begin{aligned}
\oint \frac{\partial \Delta \omega}{\partial S} d S+\Delta \theta \cdot \oint r_{s} d S \\
=\frac{1}{G} \int_{e} \Delta \tau d S+\frac{1}{G} \int_{p} \Delta \tau d S \\
\quad-\frac{1}{E} \int_{p} \frac{3 \tau}{\sigma} \Delta \sigma d S+\int_{p} \frac{3 \tau}{\sigma} \Delta \varepsilon d S
\end{aligned}
$$

where the suffixes $e$ and $p$ denote an integration over the elastic and plastic zones respectively. Since the warping of closed cross-section must be continuous at each moment, the first term of the left hand side of Eq. (10) will vanish ${ }^{13}$. Substituting Eqs. (3) and (7) into Eq. (10) and considering a uniform shear flow $\Delta q=t \cdot \Delta \tau$ yield

$$
\begin{aligned}
\Delta \theta \oint r_{s} d S \\
=\frac{\Delta q}{G} \oint \frac{d S}{t}+\frac{\Delta q}{E} \int_{p}\left(\frac{3 \tau}{\sigma}\right)^{2} \cdot \frac{d S}{t}+\Delta \varepsilon_{0} \int_{p} \frac{3 \tau}{\sigma} d S \\
\quad-\Delta \Psi_{z} \int_{p} \frac{3 \tau}{\sigma} \cdot y d S+\Delta \Psi_{y} \int_{p} \frac{3 \tau}{\sigma} z d S .
\end{aligned}
$$

Then,

$$
\begin{aligned}
\Delta q= & \frac{-1}{C_{1}+C_{2}} \\
& \times\left(C_{3} \cdot \Delta \varepsilon_{0}-C_{4} \cdot \Delta \Psi_{z}+C_{5} \cdot \Delta \Psi_{y}+C_{6} \cdot \Delta \theta\right)
\end{aligned}
$$

where

$$
\left.C_{1}=\frac{1}{G} \oint \frac{d S}{t}, \quad C_{2}=\frac{1}{E} \int_{p}\left(\frac{3 \tau}{\sigma}\right)^{2} \frac{d S}{t}\right)
$$

where

$$
\begin{aligned}
\Delta \boldsymbol{f} & =\left[\begin{array}{llll}
\Delta P & -\Delta M_{z} & \Delta M_{y} & \Delta T
\end{array}\right]^{T} \\
\Delta \boldsymbol{d} & =\left[\begin{array}{llll}
\Delta \varepsilon_{0} & -\Delta \Psi_{z} & \Delta \Psi_{y} & \Delta \theta
\end{array}\right]^{T} \\
\boldsymbol{k}_{1} & =\left(\begin{array}{cccc}
E A & E S_{z} & E S_{y} & 0 \\
E I_{z} & E I_{y z} & 0 \\
\text { sym. } & E I_{y} & 0 \\
{ }^{2} & G J
\end{array}\right) \\
\boldsymbol{k}_{2} & =\left(\begin{array}{cccc}
\frac{C_{3}^{2}}{C_{1}+C_{2}}-D_{1} & \frac{C_{3} C_{4}}{C_{1}+C_{2}}-D_{2} & \frac{C_{3} C_{5}}{C_{1}+C_{2}}-D_{3} & \frac{C_{3} C_{6}}{C_{1}+C_{2}} \\
& \frac{C_{4}^{2}}{C_{1}+C_{2}}-D_{4} & \frac{C_{4} C_{5}}{C_{1}+C_{2}}-D_{5} & \frac{C_{4} C_{6}}{C_{1}+C_{2}}
\end{array}\right)
\end{aligned}
$$

and $t=$ thickness of plate.

If none of plastic zone exists in the cross section, Eq. (12) coincides with the elastic equation of the Bredt-Batho' ${ }^{15)}$. That is,

$$
\Delta q=\frac{\oint r_{s} d S}{\oint \frac{d S}{t}} G \Delta \theta .
$$

The incremental equations of equilibrium are:

$$
\begin{aligned}
\Delta P & =\int_{e} \Delta \sigma d A+\int_{p} \Delta \sigma d A \\
& =E \int_{e} \Delta \varepsilon d A-\int_{p} \frac{3 \tau}{\sigma} \Delta \tau d A \\
\Delta M_{z} & =-\int_{e} \Delta \sigma y d A-\int_{p} \Delta \sigma y d A \\
& =-E \int_{e} \Delta \varepsilon y d A+\int_{p} \frac{3 \tau}{\sigma} \Delta \tau y d A \\
\Delta M_{y} & =\int_{e} \Delta \sigma z d A+\int_{p} \Delta \sigma z d A \\
& =E \int_{e} \Delta \varepsilon z d A-\int_{p} \frac{3 \tau}{\sigma} \Delta \tau z d A \\
\Delta T & =\oint_{\Delta \tau r_{s} d A}
\end{aligned}
$$

in which $\Delta P=$ increment of axial force; $\Delta M_{z}, \Delta M_{y}$ $=$ increments of bending moments about $z$ and $y$ axis; $\Delta T=$ increment of torsional moment; and $\int_{e} d A, \int_{p} d A=$ integrations about elastic area and plastic area. Substituting Eqs. (3) and (12) into Eq. (15) yields the tangent stiffness matrix of a cross section as follows:

$$
\Delta f=\left[k_{1}+k_{2}\right] \cdot \Delta d
$$




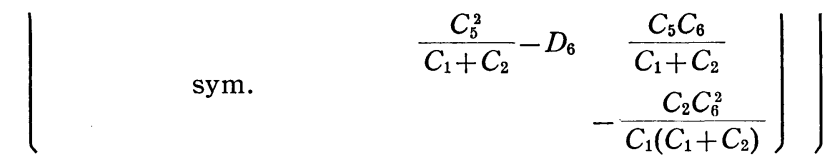

$$
\begin{aligned}
& A=\int d A, \quad S_{z}=\int y d A, \quad S_{y}=\int z d A, \\
& I_{z}=\int y^{2} d A, \quad I_{y z}=\int y z d A, \quad I_{y}=\int z^{2} d A, \quad J=\frac{\left(\oint r_{s} d S\right)^{2}}{\oint \frac{d S}{t}}, \\
& D_{1}=\int_{p} d A, \quad D_{2}=\int_{p} y d A, \quad D_{3}=\int_{p} z d A, \\
& D_{4}=\int_{p} y^{2} d A, \quad D_{5}=\int_{p} y z d A, \quad D_{6}=\int_{p} z^{2} d A .
\end{aligned}
$$

When the axes $y$ and $z$ coincide with the principal axes of the cross section, the values $S_{z}, S_{y}$ and $I_{y z}$ in Eq. (18) will vanish. In Eq. (16), the matrix $\boldsymbol{k}_{1}$ is an ordinary elastic stiffness matrix and the matrix $\boldsymbol{k}_{2}$, which consists of the terms of integration over the plastic zone, plays a role in the reduction of stiffness due to yielding.

\section{(3) Numerical Procedure}

Since no closed form solution has been possible, it is inevitable that the elements in the tangent stiffness matrix derived above are evaluated numerically by dividing the cross section into discrete sub-elements as often used in the elasto-plastic analysis ${ }^{12), 17), 22), 25) . ~ T h o u g h ~ a b o v e ~}$ stiffness matrix was formulated by means of the flow theory, numerical results based on the deformation theory can be also obtained using the fact that the prediction of the flow theory will coincide with that of the deformation theory in the case of proportional loading. A brief flow chart of the computation is shown in Appendix. The iterative procedure is executed as follows:

1) An increment of deformations $\Delta d$ is computed for an assumed increment of external forces $\Delta \boldsymbol{f}$.

2) Calculate corresponding increment of stresses at each sub-element.

3) Check the condition of the yielding and elastic unloading and distinguish the plastic subelements from the elastic sub-elements for the next loading step.

4) If some sub-elements will attain to the yield stress state in the midst of a force increment, the force and the deformation which are just great enough to cause yielding of those subelements are considered to be effective ${ }^{18}$.

5) The above steps are repeated until $\sum \Delta f$ equals a specified $\boldsymbol{f}$ in value or until fully plastic state.
By means of this iterative procedure, it is possible to analyze an elasto-plastic behavior of a member subjected to axial force, biaxial bending and uniform torsion. Numerical examples shown later are about the test specimens subjected to axial compression and uniform torsion. The number of the divided sub-elements are 48 .

\section{EXPERIMENTAL STUDY}

\section{(1) Test Specimen}

The test specimens were classified into four series ST, SB, HT and $\mathrm{HB}$ according to the materials and fabrications as shown in Table 1. The materials used herein have a stress-strain curve which may be approximately represented by an idealized elastic-perfectly plastic one within tested range of strain. The nominal dimensions were determined not to collapse by column buckling as well as local buckling until a fully plastic stress state would be reached. All specimens were not annealed, however test specimens

Table 1 Nominal Dimensions and Mechanical Properties

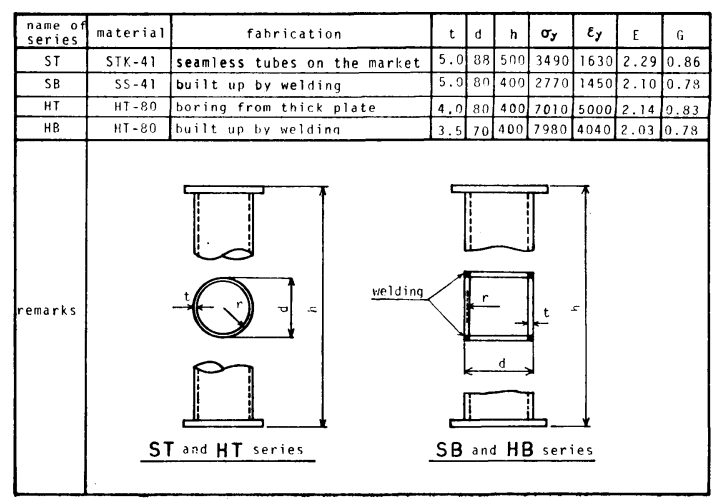


of series ST and HT may be still considered to have none of residual stresses. Longitudinal residual stresses of the specimens in series SB and HB, which were built-up by welding, were measured by the sectioning method with Hüggenberger contact-type strain-measuring apparatus. For instance, the distributions of residual stresses were obtained as shown in Fig. 2 including an idealized pattern of its distribution. Thus, the maximum compressive residual stress is nearly estimated at $0.4 \sigma_{y}$ for SB-series and $0.25 \sigma_{y}$ for $\mathrm{HB}$ series.

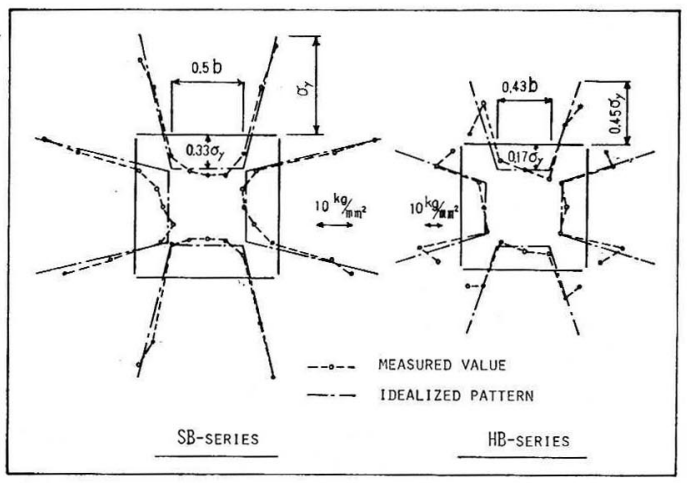

Fig. 2 Residual Stress Distributions.

\section{(2) Testing Apparatus ${ }^{23)}$}

Testing apparatus is illustrated in Figs. 3 and 4. Both compressive force and torsional force were applied by hydraulic jacks. Two equal and parallel forces with mutually opposite directions were converted to a torsional moment through a circular turn table made of thick steel plate. Both normal strain and shear strain were measured by wire strain gages bonded at the midheight cross section. Average values of four or eight strain gages were used as measured values of normal strain or shear strain of the specimens. Two calibrated load transducers, one of which was for compression and the other for torsion,

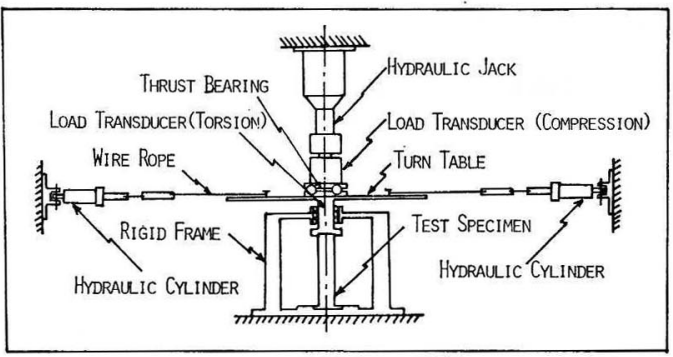

Fig. 3 Combined Compression and Torsion Testing Apparatus.

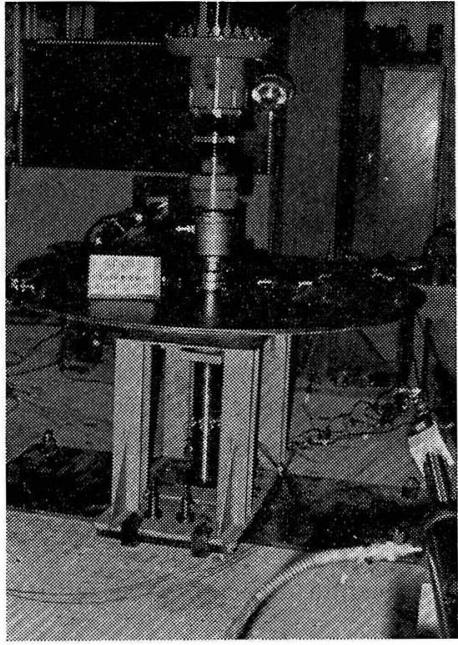

Fig. 4 Testing Apparatus under Experiment.

were used to determine and control the applied combined forces. The forces are monitored and recorded in a $\mathrm{X}-\mathrm{Y}$ recorder and a magnetic data recorder together with the deformations simultaneously.

\section{(3) Test Procedure}

At first, a test specimen was carefully set to obtain fairly uniform distribution of strains under preliminary loading of compression and torsion, independently. Then, the combined forces were increased stepwise until the proportional limit of stress was attained, and thereafter increased continuously and slowly until an ultimate state was attained. The loading was finally stopped at the state that the strains increase without any increment of force. The strain measurement was restricted to the range where the plastic and

Table 2 Loading Conditions

\begin{tabular}{|c|c|c|c|c|c|c|c|}
\hline \multicolumn{2}{|c|}{$\begin{array}{l}\text { Speci- } \\
\text { men }\end{array}$} & \multirow{2}{*}{$\begin{array}{c}\begin{array}{c}\text { Load- } \\
\text { ing }\end{array} \\
\text { P.L. }\end{array}$} & \multirow{2}{*}{$\begin{array}{c}\begin{array}{c}\text { Stress } \\
\text { Ratio }\end{array} \\
\tau=0.5 \sigma\end{array}$} & \multicolumn{2}{|c|}{$\begin{array}{l}\text { Speci- } \\
\text { men }\end{array}$} & \multirow{2}{*}{$\begin{array}{c}\begin{array}{c}\text { Load- } \\
\text { ing }\end{array} \\
\text { P.L. }\end{array}$} & \multirow{2}{*}{$\begin{array}{c}\begin{array}{c}\text { Stress } \\
\text { Ratio }\end{array} \\
\tau=0.5 \sigma\end{array}$} \\
\hline \multirow{4}{*}{ ST } & 1 & & & \multirow{4}{*}{ HT } & 1 & & \\
\hline & 2 & P.L. & $\tau=0.3 \sigma$ & & 2 & P.L. & $\tau=0.25 \sigma$ \\
\hline & 3 & C.C. & $\boldsymbol{\sigma}=0.75 \sigma_{y}$ & & 3 & P.L. & $\tau=1.0 \sigma$ \\
\hline & 4 & C.C. & $\sigma=0.87 \sigma_{y}$ & & 4 & C.C. & $\sigma=0.7 \sigma_{y}$ \\
\hline \multirow{4}{*}{ SB } & 1 & C.C. & $\sigma=0.93 \sigma_{y}$ & \multirow{4}{*}{$\mathrm{HB}$} & 1 & C.C. & $\sigma=0.83 \sigma_{y}$ \\
\hline & 2 & P.L. & $\tau=0.24 \sigma$ & & 2 & P.L. & $\tau=0.26 \sigma$ \\
\hline & 3 & C.C. & $\sigma=0.81 \sigma_{y}$ & & 3 & C.C. & $\sigma=0.69 \sigma_{y}$ \\
\hline & 4 & P.L. & $\tau=0.43 \sigma$ & & 4 & P.L. & $\tau=0.46 \sigma$ \\
\hline
\end{tabular}

Remarks: P.L. $=$ Proportional Loading

C.C. $=$ Torsion under Constant Compression 
elastic components of the strain increments are comparable to each other. Loading conditions for each specimen are summarized in Table 2 .

\section{(4) Test Results* and Considerations}

1) Torsional Moment-Rate of Twist relation When the specimens with such residual stress as shown in Fig. 2 are subjected to torsion and axial compression, the yielding first begins at the middle of each component plate and then spreads to the whole cross section with the increase of load. In order to show the influence of the residual stress, the test results of ST and SB are illustrated in Fig. 5. The pattern of the curves in Fig. 5 shows that the residual stress reduces the torsional rigidity considerably after elastic limit. Test results are compared with the theo-

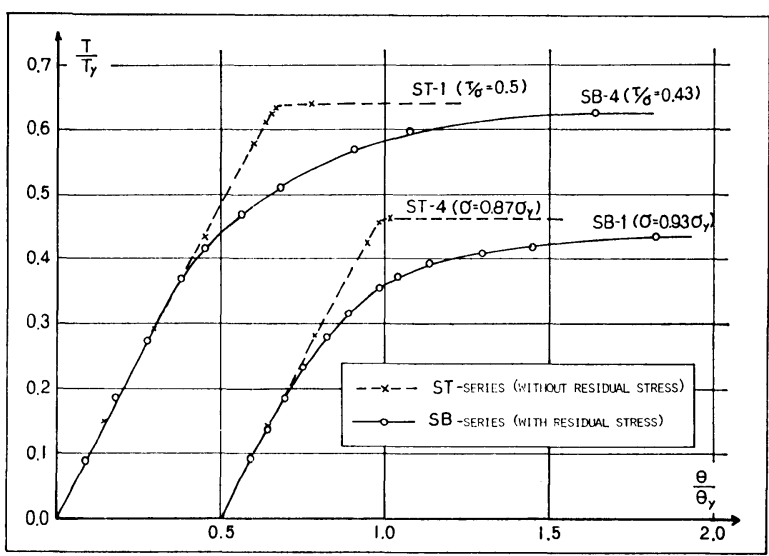

Fig. 5 Influences of Residual Stress on Torsion.

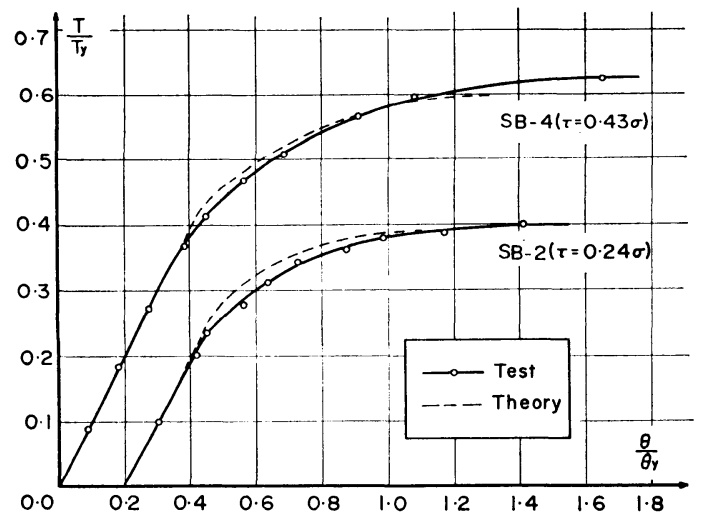

Fig. 6 Relationship between Torsion and Rate of Twist (P.L.).

* Test results shown here are mainly about SB-series and detailed data of other test series are appeared in Ref. 19.

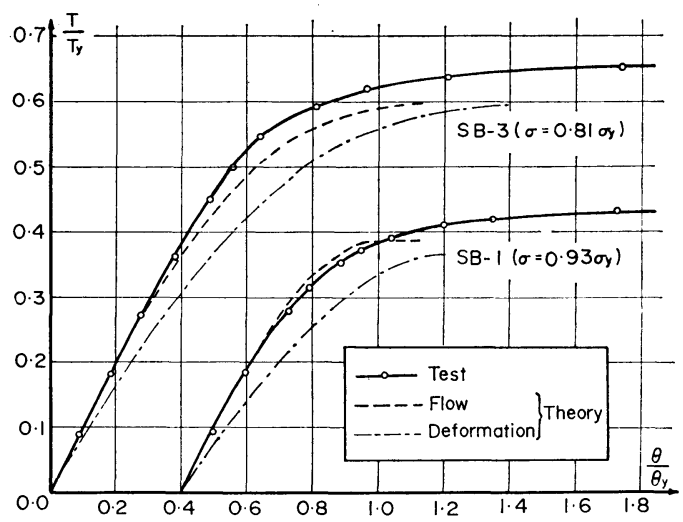

Fig. 7 Relationship between Torsion and Rate of Twist (C.C.).

retical estimations in Figs. 6 and 7, where it should be noted that the specimens SB-1 and SB-3 have partially yielded zone due to axial compression already before they receive the torsional moment. The difference between the prediction of the flow theory and that of the deformation theory appears in the case of torsion under constant compression (Fig. 7), and the former coincides with the test result more closely than the latter.

\section{2) Tangent Torsional Rigidity}

The slope of the curves in Figs. 5, 6 and 7 may be considered to be equivalent to the tangent torsional rigidity $(G J)_{t}$ and its variation concerning SB-series are shown in Fig. 8. The curves in Fig. 8 show two different shape patterns according to the two types of loading conditions, but the theoretical estimations based on the flow theory are in good agreement with the test results. It is noteworthy to point out that the initial torsional rigidities of SB-1 and SB-3, which have partially yielded zones, remains unaltered at their elastic value as predicted by the flow theory (Table 3 ). In Table 3 , it will be recognized that the prediction of the deformation theory may be a func-

Table 3 Initial Torsional Rigidity

\begin{tabular}{|c|c|c|c|c|c|c|}
\hline \multirow{3}{*}{ Specimen } & \multicolumn{3}{|c|}{$\begin{array}{l}\text { State at } \\
\text { the beginning of } \\
\text { Torsion }\end{array}$} & \multirow{2}{*}{\multicolumn{3}{|c|}{$\begin{array}{c}\text { Initial Torsional } \\
\text { Rigidity } \\
(G J)_{t} / G J\end{array}$}} \\
\hline & \multirow{2}{*}{$P / P_{y}$} & \multicolumn{2}{|c|}{$\varepsilon_{0} / \varepsilon_{y}$} & & & \\
\hline & & Theory & Test & D.T. & F.T. & Test \\
\hline SB-1 & 0.93 & 1.3 & 1.5 & 0.67 & 1.00 & 1.00 \\
\hline SB-3 & 0.81 & 1.1 & 1.2 & 0.85 & 1.00 & 1.00 \\
\hline
\end{tabular}




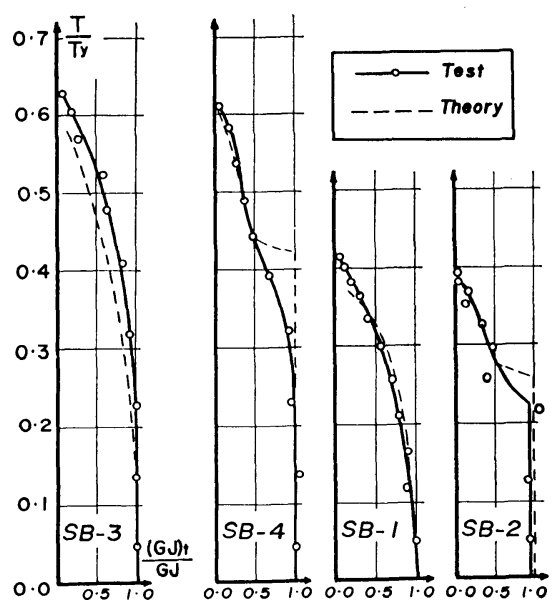

Fig. 8 Relationship between $(G J)_{t}$ and Torsion.

tion of the magnitude of plastic strain ${ }^{7)}$, but that of the flow theory will not be affected by the depth of plasticity.

3) The Variation of the Incremental Deformation Vector

Test results regarding the variation of the deformation vector, calculated as the average strain vector in the cross section, are shown in Figs. 9 and 10 including the theoretical ones calculated for the identical loading condition. The magnitude of deformation does not necessarily show good correspondence between the test results and the theoretical ones, but the following coincidence in tendency may be recognized. That is to say, in Fig. 9, with the increase of torsional moment and spread of yielded zone, the direction of the deformation vector, which has been oriented to the same direction as the force vector at the very beginning of twisting, seems to take

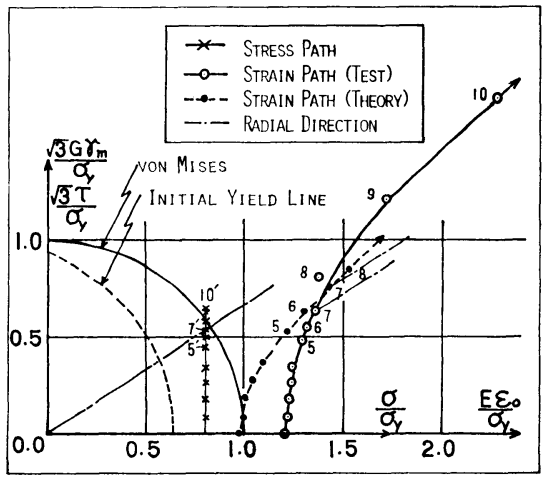

Fig. 9 Variation of Averaged Strain Vector (SB-3).

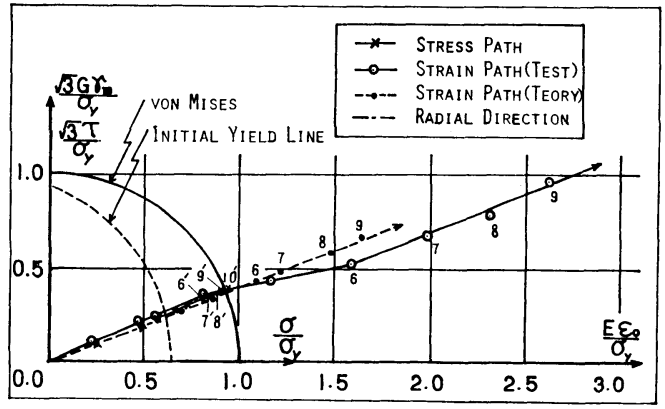

Fig. 10 Variation of Averaged Strain Vector (SB-2).

the direction of the outwardly directed normal to the yield surface as predicted by the theory based on the flow theory of plasticity. On the other hand, in the case of proportional loading, the direction of the deformation vector does not change and keeps its original one of the outwardly directed normal to the yield surface throughout loading.

4) Influences of Loading Path

One of the important differences between the flow theory and the deformation theory of plasticity is that the former is dependent on the stress and strain history and the latter is independent. In order to investigate the influences of the loading path, the deformations are calculated for four different loading paths as shown in the lower part of Fig. 11 and illustrated in Figs. 11 and 12. The flow theory as used here-

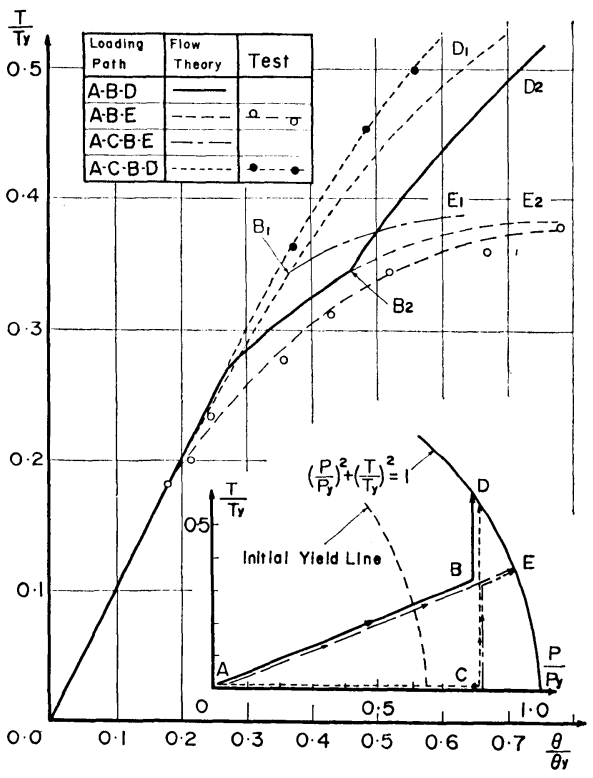

Fig. 11 Influences of Loading Path. 
in can predict two different states of deformation corresponding to the single loading condition after different loading history. That is, the curves $B_{1}-D_{1}$ and $B_{2}-D_{2}$, for example, may be predicted for the loading path B-D according to the loading histories A-C-B and A-B, respectively. While, the deformation theory will predict the only special state of deformation $B_{2}$ for the identical load condition $B$ regardless whether the loading history is A-B or A-C-B. The values of the deformations and the tangent torsional rigidity at the point $B$, where the loading path bifurcates, are summarized in Table 4. The test results about the deformation cor-

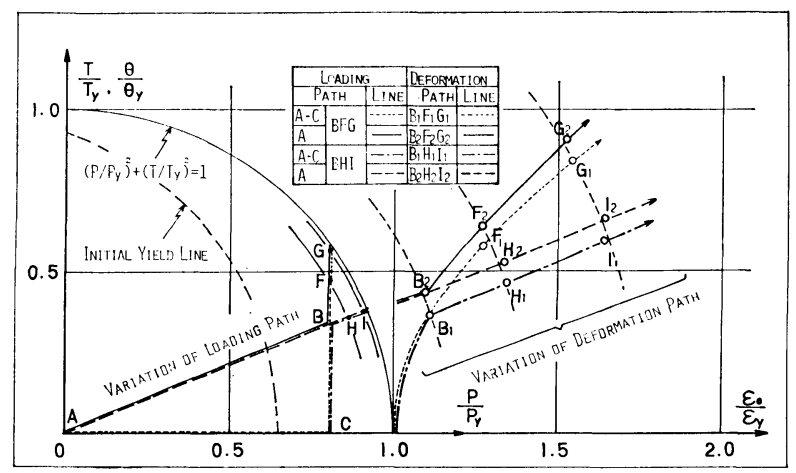

Fig. 12 Variation of Deformation Path and Loading Path.

Table 4 Deformations and Torsional Rigidity at Point B

\begin{tabular}{|c|c|c|c|c|c|c|c|c|c|c|}
\hline \multirow{3}{*}{$\begin{array}{l}\text { Loading } \\
\text { history }\end{array}$} & \multirow{3}{*}{$\begin{array}{l}\text { Direction } \\
\text { of Load } \\
\text { Increment }\end{array}$} & \multirow{2}{*}{\multicolumn{3}{|c|}{$\begin{array}{l}\text { Tangent Rigidity } \\
\qquad(G J)_{t} / G J\end{array}$}} & \multicolumn{6}{|c|}{ Deformation at B } \\
\hline & & & & & \multicolumn{3}{|c|}{$\theta / \theta_{y}$} & \multicolumn{3}{|c|}{$E \varepsilon_{0} / \sigma_{y}$} \\
\hline & & D.T. & F.T. & Test & D.T. & F.T. & Test & D.T. & F.T. & Test \\
\hline \multirow{2}{*}{ A-B } & B-D & 0.6 & 0.8 & - & \multirow{2}{*}{0.46} & \multirow{2}{*}{0.46} & \multirow{2}{*}{0.52} & \multirow{2}{*}{1.1} & \multirow{2}{*}{1.1} & \multirow{2}{*}{1.6} \\
\hline & B-E & 0.3 & 0.3 & 0.3 & & & & & & \\
\hline \multirow{2}{*}{ A-C.B } & B-D & 0.6 & 0.8 & 0.9 & \multirow{2}{*}{0.46} & \multirow{2}{*}{0.37} & \multirow{2}{*}{0.35} & \multirow{2}{*}{1.1} & \multirow{2}{*}{1.1} & \multirow{2}{*}{1.2} \\
\hline & B-E & 0.3 & 0.3 & - & & & & & & \\
\hline
\end{tabular}

Remarks: D.T.=Deformation Theory F.T. $=$ Flow Theory

responding to the load condition $B$ show not only different values according to the different loading histories, but also a significant correlation with the prediction of the flow theory. Furthermore, it can be seen that the tangent torsional rigidity at the loading state $B$ is dependent on the direction of force vector rather than the loading history*. As for the tangent torsional rigidity, the estimations of the flow theory are also in good agreement with the test results. ,Generally speaking, since the individual rigidity of the cross section, for example, the tangent torsional rigidity, $\Delta T / \Delta \theta$, is a function of other three deformations $\Delta \varepsilon_{0}$, $\Delta \Psi_{z}$ and $\Delta \Psi_{y}$ besides $\Delta \theta$, it is dependent not only on the current loading state, but also on the direction of the incremental force vector. As for the tangent torsional rigidity at $\mathrm{B}$ discussed above, the magnitude of the incremental axial force $\Delta P$

* In the former report of Ref. 19, the difference of the tangent torsional rigidity at the same loading condition misinterpreted as caused by the difference of the loading history. may have dominant effect on the magnitude of the tangent rigidity. Accordingly, the tangent rigidity of the cross section are supposed to be dependent on the manner how the redistribution of stress will occur within the cross section corresponding to the variation of the force vector. During the redistribution of stress, of course, the stress state of any point in the cross section will be in one of the state of loading, neutral-loading or unloading.

5) The Yield Criterion

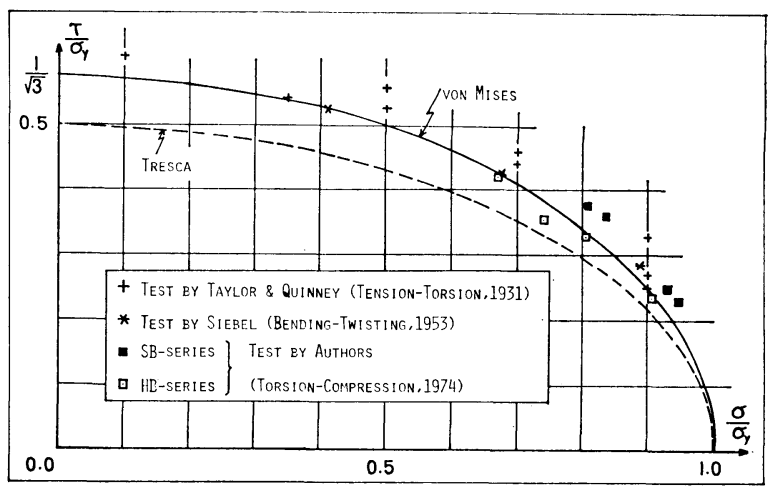

Fig. 13 Critical Stress State. 
The critical stress states, in which an overall yielding begins to occur, are shown in Fig. 13 for SB-series and HB-series together with the test results of Taylor and Quinney ${ }^{20)}$ and those of Siebel ${ }^{13)}$. The critical equivalent stresses $\sigma_{E}$ are calculated and shown in Table 5. It is recognized that the yield criterion of von Mieses can give a good approximation to all test results except HB-4 which was observed not haveing attained to fully plastic state owing to local buckling failure.

Table 5 Critical Stress State

\begin{tabular}{c|c|c|c|c}
\hline \multicolumn{2}{l|}{ Specimen } & $\sigma / \sigma_{y}$ & $\tau / \sigma_{y}$ & $\sigma_{E} / \sigma_{y}$ \\
\hline \multirow{4}{*}{ SB } & 1 & 0.929 & 0.249 & 1.024 \\
\cline { 2 - 5 } & 2 & 0.947 & 0.230 & 1.028 \\
\cline { 2 - 5 } & 3 & 0.808 & 0.377 & 1.039 \\
\cline { 2 - 5 } & 4 & 0.836 & 0.360 & 1.043 \\
\hline \multirow{3}{*}{ HB } & 1 & 0.804 & 0.330 & 0.986 \\
\cline { 2 - 5 } & 2 & 0.906 & 0.236 & 0.994 \\
\cline { 2 - 5 } & 3 & 0.669 & 0.420 & 0.999 \\
\hline & 4 & 0.739 & 0.354 & 0.960 \\
\hline
\end{tabular}

(5) Comments to Inelastic Torsional Buckling Problems

In order to account for the contradictions between the flow theory and the deformation theory, in 1949, Batdorf presented a theory for effective shear modulus based on the concept of slip as shown in Fig. 14, and justified the use of the deformation theory in the analysis of the plastic

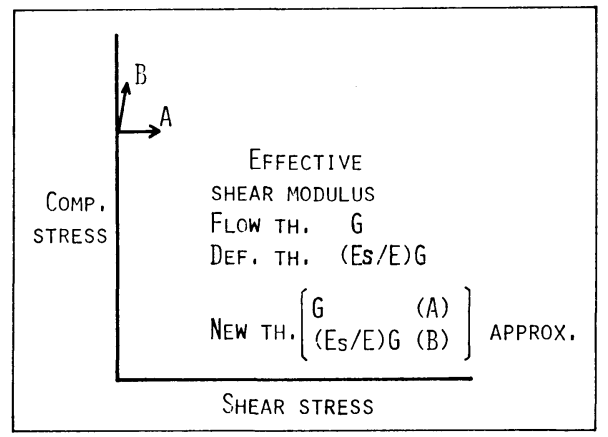

Fig. 14 Theories of Effective Shear Modulus by Batdorf. ${ }^{21)}$

buckling of plate ${ }^{21)}$. It is proposed, in his theory, that different shear modulus should be taken according to the direction of the incremental stress vector as a bifurcation problem. As for the bifurcation theory, which assumes the tor- sion under constant compression, since the tangent torsional rigidity given by the flow theory is the same as the elastic value, the buckling load predicted by the flow theory will be larger than that of actual column which necessarily have initial imperfection. If, in above case, the torsional rigidity which is predicted by the deformation theory and is smaller than the elastic value are used, a buckling load which is close to but rather smaller than the actual buckling load will be obtained. When the welded compression members collapse owing to torsional buckling failures, the initial imperfections must not be disregarded ${ }^{26)}$. In such a case, the variation of force vector composed of stress resultant $P$ and stress couple $T$ may be supposed to follow the path O-D-E as illustrated in Fig. 15. At

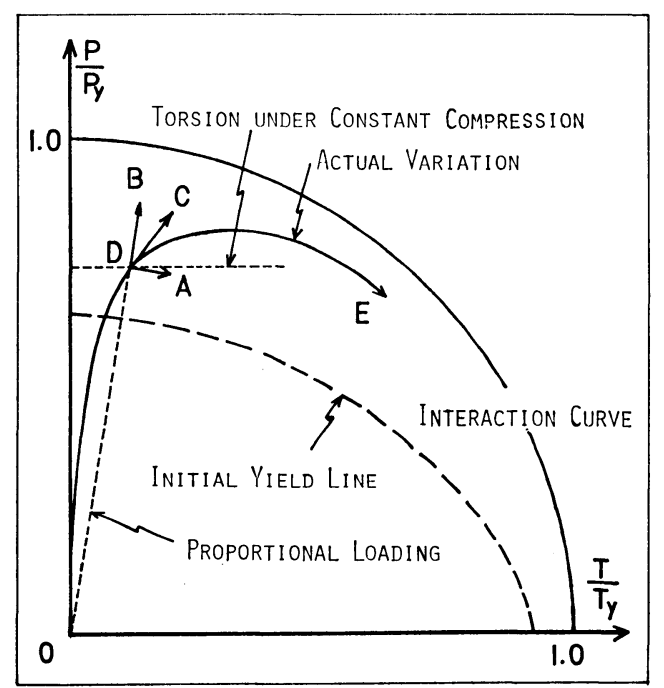

Fig. 15 Variation of Force Vector.

any stress state $D$, three particular directions of the force vector may be defined. That is, the direction $B$ is outwardly directed normal to the interaction curve, the direction $\mathrm{A}$ is normal to the line $O D$ and the direction $C$ is tangential to the supposed actual path O-D-E. The tangent torsional rigidity concerning three kinds of direction $\mathrm{A}, \mathrm{B}$ and $\mathrm{C}$ may be estimated consistently by the present method. The tangent torsional rigidity reduces its magnitude as the direction of force vector varies from $A$ to $B$. The one for $A$ will take an approximately same value with the elastic one and that for $\mathrm{B}$ will rather coincides with the prediction of the deformation theory ${ }^{24)}$. Accordingly, the concept of Batdorf about the effective shear modulus may be gene- 
ralized for a member with initial imperfection by introducing the tangent torsional rigidity based on the flow theory. The important fact about the actual behavior of the column with initial imperfection is that the column will twist with the increase of the axial compression. It is supposed, consequently, that the more close prediction of the maximum load will be obtained if the tangent torsional rigidity will be estimated by tracing the variation of the direction of the force vector by the present method. Above discussion suggests that the contradiction between the deformation theory and the flow theory in the inelastic buckling analysis may be well accounted for only by means of the flow theory, but it is necessary to show whether the actual magnitude of the initial imperfection is large enough to complete above reasoning or not ${ }^{9), 26)}$.

\section{CONCLUSIONS}

The following conclusions may be drawn from the results of this study:

1) The tangent stiffness matrix of thin-walled closed cross-section developed under some assumptions is suitable for use in elasto-plastic analysis of a tubular member with partially yielded cross-section. Then, the tangent stiffness matrix derived here may be extended and applied to the various kinds of structural analyses including torsion.

2) The theoretical estimations based on the flow theory of plasticity show good correspondence with the experimental results of welded box cross-section under combined axial compression and torsion.

3) Presence of residual stress, in general, reduces the torsional rigidity of a member.

4) The reduction of tangent torsional rigidity in elasto-plastic range is dependent on the direction of incremental force vector composed of stress resultants and stress couples besides the current stress and strain state.

5) It is confirmed experimentally again that the initial tangent torsional rigidity of the partially yielded cross-section under constant compression remains unaltered at its elastic value.

6) It was shown experimentally that the elastoplastic deformations of such realistic magnitude as occured in actual steel members take different values according to the difference of loading path, and furthermore, a sufficient explanation to this fact was given by the presented method based on the flow theory.
7) The yield criterion of von Mieses can give a good approximation to all test results regardless the loading path.

8) The possibility to account for the contradiction between the deformation and the flow theory consistently by the flow theory only was suggested from these considerations and discussions.

\section{ACKNOWLEDGEMENTS}

Part of this study was supported by the Funds for the Science and Technology from the Ministry of Education. Numerical computations were carried out at the computer center of Kumamoto University. Sincere appreciation is expressed to all the staffs of the Structural Laboratory of Osaka University for the valuable assistances shown through the experiments, in particular, to graduate student $\mathrm{K}$. Kobayashi.

\section{REFERENCES}

1) Hohenemser, K. and W. Prager: Beitrag zur Mechanik des Bildsamen Verhaltens von Flusstahl, ZAMM, B. 12, H. 1, pp. 1-14, Feb., 1932.

2) Morrison, J. L. M. and W. M. Shepherd: An experimental investigation of plastic stress-strain relations, Proc. Inst. of Mech. Eng., Vol. 163, pp. 1-9, 1950.

3) Budiansky, B., N. F. Daw, R. W. Peters and R. P. Shepherd: Experimental studies of polyaxial stress-strain laws of plasticity, Proc. of the First National Congress of Appl. Mech., ASME, pp. 503-512, June, 1951.

4) Yamada, Y.: Mechanics in Plasticity, Nikkankogyo-Shinbunsha, pp. 71, 1965. (in Japanese)

5) Handelman, G., C. Lin and W. Prager: On the mechanical behavior of metals in the strain-hardening range, Quat. Jour. of Appl. Mech., Vol. 4, No. 4, pp. 397, Jan., 1947.

6) Bijlaard, P. P.: Theory of plastic buckling of plates, Jour. of Appl. Mech., Vol. 23, No. 1, pp. 27, 1956.

7) Bijlaard, P. P.: Theory and tests on the plastic stability of plates and shells, Jour. of the Aero. Sciences, pp. 529-541, Sept., 1949.

8) Haaijer, G.: Plate buckling in the strainhardening range, Jour. of the EM, Proc. of ASCE, EM2, pp. 212, Apr., 1957.

9) Lay, M. G.: Flange local buckling in wide- 
flange shapes, Jour. of the ST., Proc. of ASCE, ST6, pp. 95-116, Dec., 1965.

10) Hill, R.: The Mathematical Theory of Plasticity, The Clarendon Press, Oxford, 1950. (translated into Japanese by $\mathrm{K}$. Washizu et al.)

11) Neal, B. G.: The lateral instability of yielded mild steel beams of rectangular crosssection, Philo. Trans., Royal Society of London, Vol. 242 (A), pp. 197-242, Jan., 1950.

12) Santathadaporn, S. and W. F. Chen: Analysis of biaxially loaded columns, Fritz Eng. Labo. Report No. 331.12, Lehigh Univ., Sept., 1970.

13) Hill, R. and M. P. L. Siebel: On combined bending and twisting of thin tubes in the plastic range, Philo. Magazine, Vol. 42, pp. 722-733, 1951.

14) Siebel, M. P. L.: The combined bending and twisting of thin cylinders in the plastic range, Jour. of the Mech. and Physics of Solids, Vol. 1, pp. 189-206, 1953.

15) Usami, T.: Inelastic behavior of thin-walled open sections under combined axial force, bending and twisting, Proc. of the JSCE, No. 220, pp. 9-16, Dec., 1973. (in Japanese)

16) Komatsu, S.: Theory and Calculation of Thin Walled Structures I, pp. 125, Sankaido, 1969. (in Japanese)

17) Parikh, B. P.: Elastic-plastic analysis and design of unbraced multi-story steel frames, Fritz Eng. Labo. Report No. 273.44, Lehigh Univ., May, 1966.

18) Yamada, Y., N. Yoshimura and T. Sakurai: Plastic stress-strain matrix and its application for the solution of elastic-plastic problems by the finite element method, Int. Jour. of Mech. Science, Vol. 10, pp. 343-354, 1968.

19) Komatsu, S. and T. Sakimoto: Elasto-plastic behavior of steel members under combined torsion and compression, Tech. Report of the Osaka Univ., Vol. 23, No. 1149, pp. 681-695, 1973.

20) Taylor, G. I. and H. Quinney: The plastic distorsion of metals, Philo. Trans., Royal Society of London, Vol. 230 (A), pp. 323-362, 1931.

21) Batdorf, S. B.: Theories of plastic buckling, Jour. of the Aero. Sciences, Vol. 16, No. 7, pp. 405-408, July, 1949.

22) Fujita, Y., K. Yoshida and T. Ohkatsu: Elasto-plastic analysis of column subjected to bi-axial bending, Jour. of the Society of Naval Arch. of Japan, No. 126, pp. 217-226, No. 127 , pp. $143-150,1970$. (in Japanese)
23) Farwell, Jr., C. R. and T. V. Galambos: Nonuniform torsion of steel beams in inelastic range, Jour. of the ST., Proc. of ASCE, ST12, pp. 2813-2829, Dec., 1969.

24) Nishino, F. and L. Tall: Residual stress and local buckling strength of steel columns, Proc. of JSCE, No. 172, pp. 79-96, Dec., 1969.

25) Yoshida, H. and S. Nishida: Lateral-torsional properties of wide-flange sections with residual stresses, Memoirs of the Faculty of Technology, Kanazawa Univ., Vol. 6, No. 3, pp. 13-23, 1971.

26) Onat, E. T. and D. C. Drucker: Inelastic instability and incremental theory of plasticity, Jour. of the Aero. Sciences, Vol. 20, No. 3, pp. 181-186, Mar., 1953.

\section{NOTATIONS}

$A=$ cross sectional area;

$b \quad=$ width of component plates of the cross section;

$C_{1} \sim C_{6}=$ component of tangent stiffness matrix defined in Eq. (13);

$D_{1} \sim D_{6}=$ component of tangent stiffness matrix defined in Eq. (18);

$\boldsymbol{d}=$ vector of deformation;

$E \quad=$ modulus of elasticity;

$E_{s} \quad=$ secant modulus of elasticity;

$\boldsymbol{f}=$ vector of force;

$G \quad=$ shear modulus;

$G J=$ torsional rigidity;

$(G J)_{t}=$ tangent torsional rigidity;

$I_{y}, I_{z}=$ moment of inertia of the cross section about $y$ and $z$;

$I_{y z} \quad$ =product of inertia of the cross section;

$J=$ St. Venant torsion constant for a closed cross section;

$\boldsymbol{k}_{1}, \boldsymbol{k}_{2}=$ tangent stiffness matrix;

$M_{y}, M_{z}=$ bending moment about $y$ and $z$;

$P \quad=$ axial force;

$P_{y} \quad=$ fully plastic axial force;

$q \quad=$ shear flow;

$r_{s}=$ normal distance from the shear center to a tangent at any point of the cross section;

$s \quad=$ curvilinear coordinate along component plates of the cross section;

$S_{y}, S_{z}=$ moment of area of the cross section about $y$ and $z$;

$T \quad=$ torsion;

$T_{y}$ =fully plastic torsion; 
$t \quad=$ thickness of component plates of the cross section;

$u=$ displacement of the origin of the coordinates in the $z$ direction;

$v, w=$ displacements of the section's shear center in $x$ and $y$ direction, respectively;

$x, y, z=$ coordinate system;

=shear strain;

= normal strain;

$\varepsilon_{0} \quad=$ normal strain at the origin of the coordinates;

$\varepsilon_{r} \quad=$ residual strain due to welding;

$\varepsilon_{y} \quad=$ yield strain;

$\Psi_{y}, \Psi_{z}=$ curvature about $y$ and $z$;

$\theta \quad=$ rate of twist;

$\theta_{y}=$ rate of twist at the beginning of fully plastic state due to torsion;

$\sigma \quad=$ normal stress;

$\sigma_{E}=$ equivalent stress defined as $\sqrt{\sigma^{2}+3 \tau^{2}}$;

$\sigma_{y} \quad=$ yield stress;

$\tau \quad=$ shear stress;

$\phi \quad=$ angle of twist;

$\omega=$ warping.

\section{APPENDIX}

Flow Chart for Numerical Computation

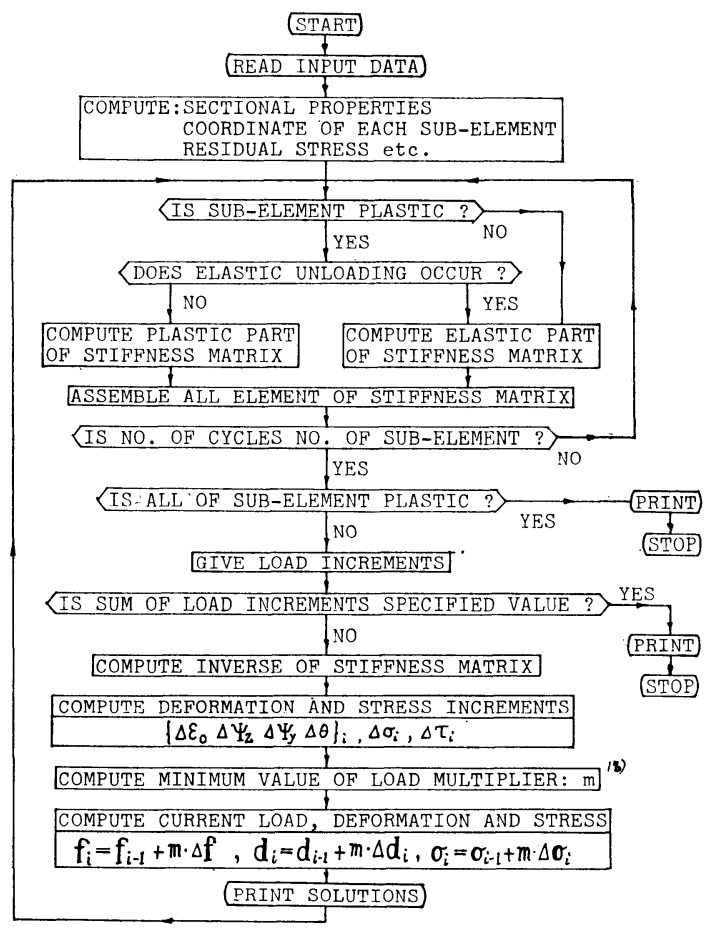

(Received July 14, 1974) 